Eur Biophys J (1989) 17:53-59

\title{
Quantal charge redistributions accompanying the structural transitions of sodium channels
}

\author{
F. Conti ${ }^{1 *}$ and W. Stühmer ${ }^{2}$ \\ 1 Istituto di Cibernetica e Biofisica, CNR, I-16146 Genova, Italy \\ 2 Max-Planck-Institut für biophysikalische Chemie, Abteilung Membranbiophysik, Am Fassberg, D-3400 Göttingen, \\ Federal Republic of Germany
}

Received February 20, 1989/Accepted in revised form March 17, 1989

\begin{abstract}
Asymmetric displacement currents, $I_{g}$, associated with the gating of nerve sodium channels have been recorded in cell-attached macropatches of Xenopus laevis oocytes injected with exogenous mRNA coding for rat-brain-II sodium channels. The $I_{g}$ properties were found to be similar to those of gating currents previously observed in native nerve preparations. $I_{g}$ fluctuations were measured in order to ascertain the discreteness of the conformational changes which precede the channel opening. The autocorrelation of the fluctuations is consistent with a shot-like character of the elementary $I_{g}$ contributions. The variance of the fluctuations indicates that most of the gating-charge movement that accompanies the activation of a single sodium channel occurs in 2 to 3 brief packets, each carrying an equivalent of about 2.3 electron charges.
\end{abstract}

Key words: Sodium channel, gating current, fluctuation analysis, patch clamp

\section{Introduction}

The permeability changes that regulate many cellular functions, in particular electrical excitation, result from random openings and closures of ionic channels driven by thermal motion and modulated by environmental factors, e.g. the membrane potential, which affect the relative probability of the channels being open or closed. The first evidence of the stochastic nature of ion-channel events was provided by fluctuation analysis (Katz and Miledi 1970; Conti et al. 1975; Conti and Wanke 1975; Neher and Stevens 1977). Single-channel recordings (Neher and Sakmann 1976; Hamill et al. 1981) directly demonstrated the discreteness of the open-close transition, which is practically instantaneous on the microsecond time scale. It has since been a common practice to model ion channels using kinetic schemes with a finite number of statistically signifi- cant states and to assume Markovian transitions that are instantaneously voltage dependent (French and Horn 1983). Within such schemes the electrophysiological parameters can be related to the thermodynamic properties of the various channel conformations (Schwarz 1978; Conti 1986).

The transitions which precede the opening of a channel cannot be viewed directly by patch-clamp recordings and only limited inferences can be made by analysis of single-channel records (Horn and Vandenberg 1984). For the sodium channels of nerve the existence of several closed conformations is a necessary condition imposed by the multiexponential time course of the macroscopic currents (Conti and Wanke 1975) and by the fact that the charge redistributions which accompany the operation of the channels (gating currents) largely precede the actual opening (Armstrong and Bezanilla 1974; Keynes and Rojas 1976). However, there is so far no direct evidence for a small number of closed states implied by a literal translation of the Hodgkin-Huxley $(\mathrm{HH})$ equations (Hodgkin and Huxley 1952) into a kinetic scheme (FitzHugh 1965). Indeed, several authors have proposed models which assume a continuum of possible charge distributions within the channel protein (Neumcke et al. 1976; Edmonds 1987).

Continuous and discrete models lead to qualitatively different expectations for the gating current that one could ideally measure at the single channel level. In the continuous case one expects to observe singlechannel events with finite amplitude and a time course similar to that of the mean gating current, $I_{g}$, obtained in the presence of a large number of channels. Discrete models predict instead the occurrence of few needle pulses, synchronous with the various transitions which involve a major charge redistribution.

In practice, a single "shot" of charge, $q$, occurring at time, $t^{\prime}$, will appear as a smooth current signal, $i(t)=q h\left(t-t^{\prime}\right)$, where $h(t)$ is the impulse response of the recording apparatus related to the effective band- 
width, $B$, by:

$B=\frac{1}{2} \int_{0}^{\infty} h^{2}(t) \mathrm{d} t$

For an 8-pole Bessel filter $B$ is very close to the 3 -dB cut-off frequency. $h(t)$ is a bell-shaped function that has a half width of about $1 /(2 B)$ and reaches a peak value of about $2 \mathrm{~B}$ with a delay $\theta=1 / B$. When viewed through such a filter with $B=10 \mathrm{kHz}$, a "shot" of 1 electron charge $e_{0}=1.6 \cdot 10^{-19} \mathrm{C}$ would appear as a current signal with about $3 \mathrm{fA}$ amplitude and about $50 \mu$ s width. This current is about 100 times too small to be resolved with present patch-clamp technology, but its quantal nature may still be revealed by the analysis of macroscopic fluctuations.

We report here measurements of gating-current noise in cell-attached macropatches of Xenopus laevis oocytes injected with exogenous mRNA coding for rat-brain-II sodium channels (Noda et al. 1986; Stühmer et al. 1987). This preparation provides excellent recordings of apparently normal sodium currents in very low background noise conditions (Stühmer et al. 1987). A brief report of the experimental results has appeared (Conti and Stühmer 1988). The underlying theory is particularly simple for unidirectional gating currents, as those expected following saturating step depolarizations, or step repolarizations to large negative potentials.

\section{Theory}

Let $p\left(t^{\prime}\right)$ be the probability density that a gating transition translocating a charge $q_{j}$ occurs at time $t^{\prime}$. Provided $p(t)$ varies slowly in comparison to $h(t)$, the mean and the mean square of the "single-gate" current observable in repeated trials will be given by:

$$
\begin{aligned}
& \left\langle i_{j}(t)\right\rangle=q_{j} \int_{0}^{t} h\left(t-t^{\prime}\right) p\left(t^{\prime}\right) \mathrm{d} t \approx q_{j} p(t-\theta) \\
& \left\langle i_{j}^{2}(t)\right\rangle=q_{j}^{2} \int_{0}^{t} h^{2}\left(t-t^{\prime}\right) p\left(t^{\prime}\right) \mathrm{d} t \approx 2 B q_{j}^{2} p(t-\theta)
\end{aligned}
$$

and the variance of such measurements, $\sigma_{j}^{2}(t)=\left\langle i_{j}^{2}(t)\right\rangle-\left\langle i_{j}(t)\right\rangle^{2}$

can be expressed as:

$\sigma_{j}^{2}(t)=2 B q_{j}^{2} p(t-\theta)\left(1-\frac{p(t-\theta)}{2 B}\right)$.

For $N$ independent single-gate events the mean, $I_{g}$, and the variance, $\sigma_{g}^{2}(t)$, of the total gating current are given by:

$$
\begin{aligned}
& I_{g}(t)=\sum_{1}^{N} q_{j} p(t-\theta) \\
& \sigma_{g}^{2}(t)=\sum_{1}^{N} \sigma_{j}^{2}(t) .
\end{aligned}
$$

If each event is characterized by the same $p(t)$ :

$I_{g}(t)=Q p(t-\theta)$

$\sigma_{g}^{2}(t)=2 B z_{a} e_{0} I_{g}(\mathrm{t})\left(1-\frac{I_{g}(t)}{2 B Q}\right)$,

where:

$Q=\sum_{1}^{N} q_{j}$

is the total gating charge, and where:

$z_{a} e_{0}=\left(\sum_{j} q_{j}^{2}\right) / Q$

is an apparent single-gate charge that coincides with the charge actually carried by each single-gate event when the $q_{j}$ 's are all identical. More generally, suppose that $I_{g}(t)$ is made of contributions from independent identical channels, but several steps carrying different charges, $z_{1} e_{0}, z_{2} e_{0}, \ldots, z_{n} e_{0}$, contribute to the total charge movement, $z e_{0}$, occurring within each single channel. Then, the apparent single-gate valence in Eq. (4) is given by:

$z_{a}=\frac{\sum_{k=1}^{n} z_{k}^{2}}{z}$.

In this case $z_{a}$ depends on the partition of $z$ among different steps, and it can only be stated that at least one of the $z_{i}$ 's exceeds or equals $z_{a}$, while none of them can exceed $\sqrt{z z_{a}}$.

Apart from the generalization to transient phenomena involving a finite total charge transfer, Eq. (4) is similar to that of classical shot noise (Schottky 1918; see e.g. DeFelice 1981). It predicts that gating currents of $50 \mathrm{pA}$ resulting from quantal charge movements with $z_{a}=1$ would show root mean square fluctuations of about $400 \mathrm{fA}$ when recorded with $B=10 \mathrm{kHz}$. These fluctuations are comparable in size to the background noise of a good patch-clamp recording system.

\section{Materials and methods}

Xenopus laevis oocytes were isolated, injected with exogenous mRNA coding for rat brain type II sodium channels (Noda et al. 1986; Stühmer et al. 1987) and freed of the folicular and vitelline membrane (Methfessel et al. 1986). Currents were recorded from cell-attached macro-patches as described by Stühmer et al. (1987). The extracellular medium and the pipette filling solution for ionic current measurements were normal Ringer's solution (in $\mathrm{m} M: 115 \mathrm{NaCl}, 2.5 \mathrm{KCl}$, $1.8 \mathrm{CaCl}_{2}, 10 \mathrm{HEPES}, \mathrm{pH}$ 7.2). $I_{g}$ measurements were made in sodium-free solutions (Tris ${ }^{+}$. replacing $\mathrm{Na}^{+}$) containing in addition $1 \mu M$ tetrodotoxin (TTX). Raising TTX to $30 \mu M$ did not affect the results. The 
voltage stimulation and the acquisition of patch-currents were controlled by a computer (DEC-PDP-11/ 73) using a special purpose program in Brown-BASIC. Before being fed to the computer, the output of the patch-clamp amplifier (EPC-7, List Electronics) was filtered by an 8-pole Bessel filter (Frequency Devices LP 902). The stimulation protocol for $I_{g}$ measurements consisted of several groups of 20 repetitions of step depolarizations to various test potentials from a conditioning level of $-140 \mathrm{mV}$ kept for $12 \mathrm{~ms}$. The series began and ended with two control stimulation groups having the same temporal pattern but test potentials to $-120 \mathrm{mV}$ or $-160 \mathrm{mV}$, respectively. The responses to the control stimulations were used in the off-line analysis for subtraction of the linear component of membrane currents.

Noise measurements were obtained from the analysis of repeated trials as in the standard non-stationary fluctuation analysis of ion-channel noise (Sigworth, 1977). The collected ensembles of records consisted of alternating groups of 100 responses to test stimulations and 10 responses to control stimulations. Test records were obtained by pulsing to potentials between 0 and $20 \mathrm{mV}$ from holding potentials of $-120 \mathrm{mV}$ or $-100 \mathrm{mV}$. Control responses were obtained from an identical temporal pattern of stimulation in which the pulses consisted of $20 \mathrm{mV}$ hyperpolarizing steps. Differences between two successive test records were used for the analysis of the fluctuations (Conti et al. 1980). The mean control record was appropriately scaled and used to subtract the linear response of the patch from the mean test record to obtain an accurate measurement of the mean gating current $I_{g}$. Repeated stimulations could be applied at a rate of $5 \mathrm{~s}^{-1}$ and a minimum of 600 records were collected in each noise measurement. In the experiment shown in the Fig. 2 the voltage stimulation was a step function. In other experiments, particularly when the initial gating current exceeded $100 \mathrm{pA}$, the stimulating voltage pulse was passed through an RC filter with $100 \mu$ s time constant. This avoided saturation of the amplifier in the high-gain range which yields the lowest noise referred to input. In these cases $B$ was reduced to $5 \mathrm{kHz}$. The variance of the fluctuations, $\sigma^{2}(t)$, was estimated from the average square of difference records at any time during a stimulation cycle.

\section{Results}

\section{Macroscopic gating currents}

Figure $1 \mathrm{~A}$ shows a series of gating-current responses produced by step depolarizations to various voltages between $-100 \mathrm{mV}$ and $+40 \mathrm{mV}$ in steps of $20 \mathrm{mV}$
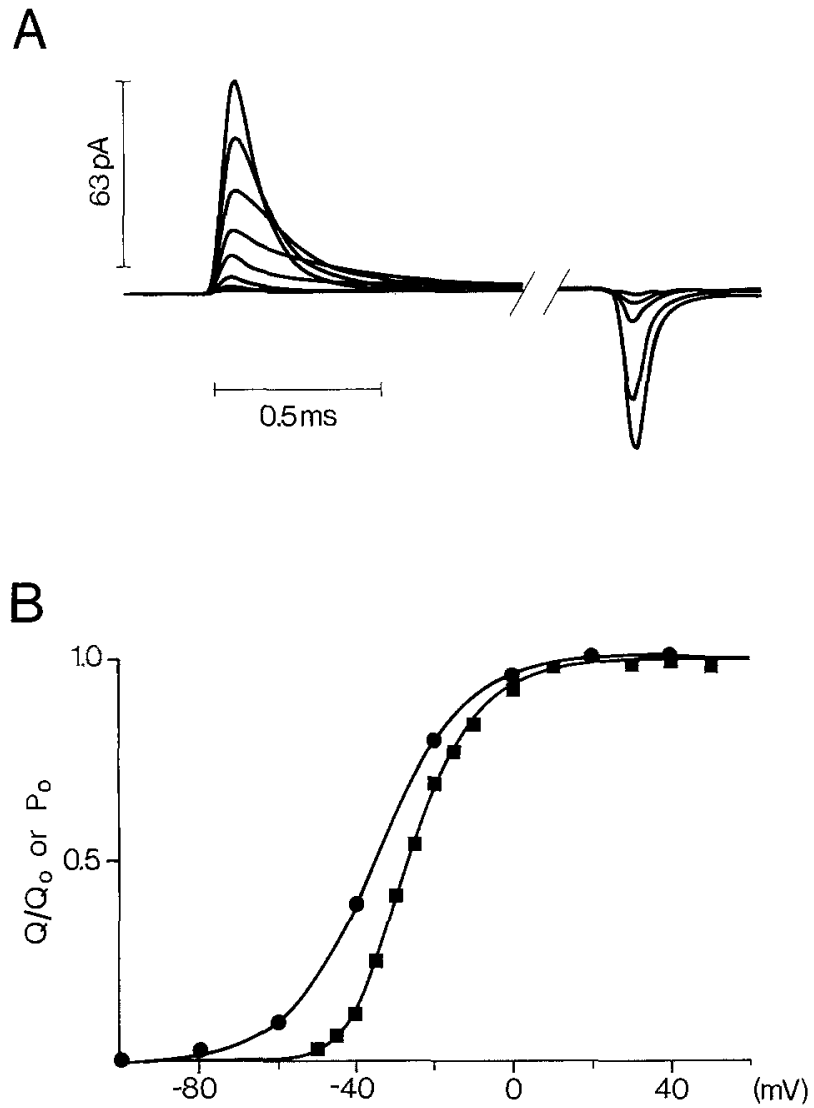

Fig. 1. A. Sodium gating currents, $I_{g}$, recorded from a membrane patch of Xenopus oocyte containing about 15,000 exogenous sodium channels. Test potentials from $-100 \mathrm{mV}$ to $+40 \mathrm{mV}$ increasing in steps of $20 \mathrm{mV}$. Recording bandwidth: $B=8 \mathrm{kHz}$. Temperature: $T=15^{\circ} \mathrm{C}$. The break in the time axis represents an interval of about $1 \mathrm{~ms}$. B Comparison of the voltage dependencies of the gating charge, $Q$, (full dots) and the activation-probability of soidum currents, $P_{o}$, (full squares). The two sets of data were obtained from different oocytes. The continuous lines show the least-squares fit of either plot according to Eqs. (6) and (7), respectively, with: $Q_{o}=16.1 \mathrm{fC}, V_{g}=-34.8 \mathrm{mV}$, $z_{g}=2.2 ;$ and $V_{m}=-40 \mathrm{mV}, z_{m}=2.4 . P_{o}$ was estimated by assuming that the extrapolation to time zero of the exponential inactivation of sodium currents gives the steady-state current, $I^{\prime}$, that would be reached in absence of inactivation. An empirical function, $f(V)$, accounting for the non-linear current-voltage relationship of sodium channels was obtained from instantaneous $I-V$ relations in tail-current measurements (Stimers et al. 1985). $I^{\prime}$ was divided by $f(V)$ and normalized to its asymptotic value at large depolarizations to yield the $P_{o}$ data

following a conditioning pulse to $-140 \mathrm{mV}$. The responses were recorded with a bandwidth of $8 \mathrm{kHz}$. Notice that the "off" responses following the four largest depolarizations overlap almost exactly. This is due both to the saturation of the "on-charge" displacement (Fig. 1 B) and to the increase in "off-charge" immobilization brought about by a more complete inactivation of the channels at the end of the larger pulses (Armstrong and Bezanilla, 1977). For each voltage, V, the total gating charge, $Q$, was measured by integrating $I_{g}(t)$ over the $2 \mathrm{~ms}$ period of the test pulse. A nor- 
A

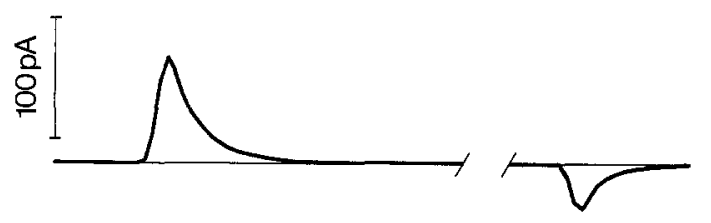

B

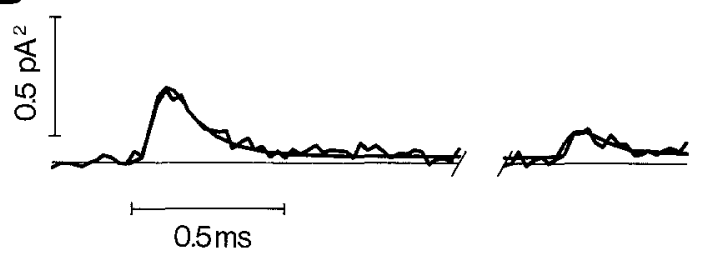

Fig. 2. The relationship between mean gating currents and their mean squared fluctuation in repeated measurements. A Average of $1.730 I_{g}$ responses to the same test depolarization at $+20 \mathrm{mV}$ from a holding potential of $-100 \mathrm{mV}$. B Noisy trace: mean square fluctuation of the $I_{g}$ recordings, offset by the mean baseline variance $\left(0.42 \mathrm{pA}^{2}\right)$; smooth trace: expected variance of the shot-noise from gating-charge quanta with an estimated valence $z_{a}=2.2$ (Eq. (4); see also Fig. 3). Notice that the same $z_{a}$, but different $Q$ 's (respectively $13.3 \mathrm{fC}$ and $4.3 \mathrm{fC}$ ), were used to draw the smooth line for the pulse and for the tail data. In $\mathbf{A}$ and $\mathbf{B}$ the break in the time axis represents an interval of about $2 \mathrm{~ms}$. Recording bandwidth: $B=8 \mathrm{kHz}$. Temperature: $T=15^{\circ} \mathrm{C}$

Table 1. Total charge, $V_{g}$ and single-shot charge

\begin{tabular}{|c|c|c|c|c|}
\hline File & $\begin{array}{l}Q_{o} \\
{[\mathrm{fC}]}\end{array}$ & & $\begin{array}{l}V_{g} \\
{[\mathrm{mV}]}\end{array}$ & $z_{g}$ \\
\hline N35 & 8.8 & & -16.7 & 2.3 \\
\hline N36A & 8.2 & & -21.5 & 2.4 \\
\hline N36B & 8.6 & & -16.1 & 2.0 \\
\hline $\mathrm{N} 47$ & 30.7 & & -13.0 & 1.8 \\
\hline N48 & 28.7 & & -17.3 & 1.7 \\
\hline N49 & 29.2 & & -17.1 & 2.2 \\
\hline N50 & 28.8 & & -20.7 & 1.8 \\
\hline Q48 & 6.0 & & -49.3 & 2.2 \\
\hline \multirow[t]{2}{*}{ N92 } & 16.1 & & -34.8 & 2.2 \\
\hline & & Mean: & $-23( \pm 12)$ & $2.07( \pm 0.25)$ \\
\hline
\end{tabular}

malized plot of $Q$ vs. $V$ is shown in Fig. $1 \mathrm{~B}$, together with a plot of the open-channel probability, $P_{o}$, estimated in a different oocyte by analysis of the macroscopic sodium currents recorded in the absence of TTX.

The gating current data of Fig. 1 are qualitatively similar to those obtained from squid axons and frog nodes of Ranvier (Armstrong and Bezanilla 1974; Keynes and Rojas 1976; Neumcke et al. 1976) except that the $Q-V$ plot apparently does not show the shoulder at potentials negative to $-60 \mathrm{mV}$, which is often found in the squid preparation (Keynes 1983; Stimers et al. 1985). The continuous lines in Fig. $1 \mathrm{~B}$ are least squares fits of $Q$ and $P_{o}$ data, respectively, according to the following equations:

$Q=Q_{o} /\left(1+\exp \left(-z_{g} e_{0} \frac{\left(V-V_{g}\right)}{k T}\right)\right)$

$P_{o}=1 /\left(1+\exp \left(-z_{m} e_{0} \frac{\left(V-V_{m}\right)}{k T}\right)\right)^{3}$

with $Q_{o}=16.1 \mathrm{fC}, V_{g}=-34.8 \mathrm{mV}, z_{g}=2.2$; and $V_{m}=$ $-40 \mathrm{mV}, z_{m}=2.4$. The values of $V_{m}$ and $z_{m}$ are close to the mean values typically found for these $\mathrm{Na}$ channels when expressed in oocytes (Stühmer et al. 1989). Values of $Q_{o}, V_{g}$ and $z_{g}$ from nine different experiments are given in Table 1.

\section{Fluctuation measurements}

Figure $2 \mathrm{~A}$ shows the average of nearly 2000 gating current responses to step depolarizations to a test potential, $V_{T}=+20 \mathrm{mV}$ from the holding potential, $V_{H}=-100 \mathrm{mV}$. The records were obtained from the same oocyte shown in Fig. 1 A with $B=8 \mathrm{kHz}$. Linear components were subtracted using appropriately scaled control responses to steps from -100 to $-120 \mathrm{mV}$. The mean records obtained from the first 200 and from the last 200 trials of the same series were not appreciably different from the overall mean, showing that the $I_{g}$ response was stable during the $7 \mathrm{~min}$ period of the whole measurement. All experiments reported here satisfied this condition of stability, which is necessary for trustworthy measurements of random fluctuations.

Figure 2 B shows the change in the fluctuation variance, $\sigma^{2}$, produced at the onset and the end of the test pulse. The relatively smooth line in Fig. $2 \mathrm{~B}$ was drawn assuming $\sigma^{2}$ to be the sum of a constant background value, $\sigma_{B}^{2}$ and a time-dependent term related to the $I_{g}$ shown in Fig. $2 \mathrm{~A}$ according to Eq. (4) with $z_{a}=2.2$. The latter value was obtained from the least squares fit of $z_{a}$ to the $\sigma_{g}^{2}-I_{g}$ relationship during the test pulse as shown in Fig. 3 .

The good fit of the simple theory to the entire time course of $I_{g}$ and $\sigma^{2}$ data justifies the assumption that partial gating-charge movements proceed independently and with similar kinetics. For voltage steps from $V_{H}$ near $-100 \mathrm{mV}$ to $V_{T}>=0$, this assumption is also supported by the observation that the decays of both the "on" and the "off" $I_{q}$ are single exponentials: in the example of Fig. $2 \mathrm{~A}$ the time constants of the best fits of these exponentials were 100 and $70 \mu \mathrm{s}$, respectively.

A most important feature of the results shown in Fig. $2 \mathrm{~B}$ is that the same $z_{a}$ fits the fluctuations of both the "on" and the "off" phase of $I_{g}$, although the charge which is free to move back during the early phase of repolarization is only one third of that displaced dur- 


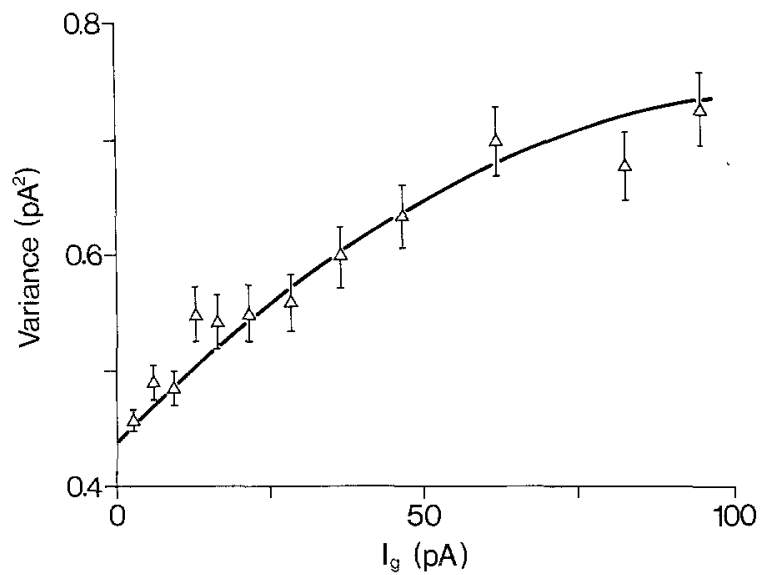

Fig. 3. Plot of the variance vs. mean data of Fig. 2 during the pulse interval starting at the apparent peak of the $I_{g}$ response; the continuous line is the least-squares fit of the variance- $I_{g}$ relation as sum of a constant background and the shot-noise of Eq. (4) with $z_{a}=2.2$. Notice that the variance axis is offset by about the baseline variance $\left(0.42 \mathrm{pA}^{2}\right)$. For convenience of display the data are shown condensed in bins obtained by dividing the $I_{g}$ values in 20 equal intervals. The error bars represent \pm standard deviations, estimated according to the measured variance taking into account both the number of trials used for estimating the mean and the variance and the number of data points in each bin

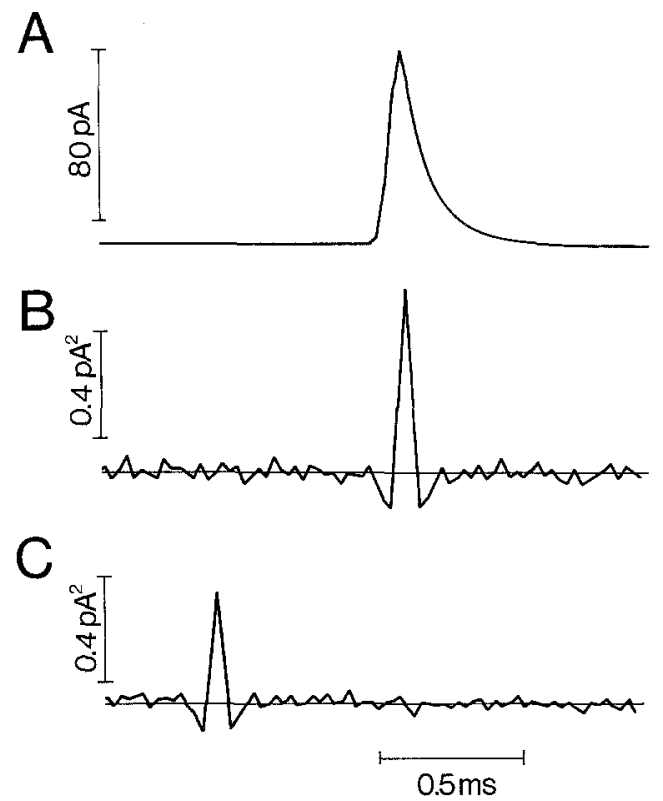

Fig. 4. A Time course of the average gating current, $I_{g}$, from the same experiment illustrated in Fig. 2, shown on an expanded time scale. B Autocovariance of the fluctuations during the development of the $I_{g}$ response. C Autocovariance of the baseline fluctuations. Each fluctuation autocovariance function was measured as the average product of the fluctuation at the respective reference time and the fluctuations at any other time of the stimulation cycle. The decay phase of $I_{g}$ is well fitted by a single exponential with a time constant of $100 \mu \mathrm{s}$, whereas both autocovariances in $\mathrm{B}$ and $\mathrm{C}$ drop to negligible values within one sampling interval $(25 \mu s)$
Table 2. Results

\begin{tabular}{lllrllllr}
\hline File & $\begin{array}{l}\text { B } \\
{[\mathrm{kHz}]}\end{array}$ & $\begin{array}{l}V_{H} \\
{[\mathrm{mV}]}\end{array}$ & \multicolumn{1}{c}{$\begin{array}{l}V_{T} \\
{[\mathrm{mV}]}\end{array}$} & $\begin{array}{l}Q \\
{[\mathrm{fC}]}\end{array}$ & $z_{a}$ & s.d. & $\begin{array}{l}\sigma_{B}^{2} \\
{[\mathrm{pA}]}\end{array}$ & N.Rec. \\
\hline N32 & 5 & -120 & 20 & 8.5 & 2.4 & 0.2 & 0.15 & 2.200 \\
N52 & 5 & -100 & 20 & 23.4 & 2.2 & 0.3 & 0.34 & 750 \\
N55 & 5 & -120 & 0 & 7.2 & 3.0 & 0.6 & 0.21 & 900 \\
N56 & 5 & -100 & 20 & 6.5 & 2.8 & 0.7 & 0.21 & 870 \\
N62 & 5 & -100 & 20 & 10.7 & 2.3 & 0.2 & 0.086 & 850 \\
N63 & 5 & -120 & 0 & 15.7 & 2.0 & 0.2 & 0.089 & 680 \\
N65 & 5 & -100 & 20 & 64.5 & 2.3 & 0.2 & 0.69 & 870 \\
N66 & 5 & -120 & 0 & 86.8 & 2.3 & 0.2 & 0.66 & 930 \\
N67 & 5 & -120 & 0 & 29.6 & 2.2 & 0.2 & 0.19 & 810 \\
K69 & 5 & -100 & 10 & 7.8 & 2.6 & 0.4 & 0.12 & 560 \\
N80 & 5 & -100 & 20 & 3.7 & 2.8 & 0.2 & 0.073 & 1.690 \\
K83 & 10 & -100 & 10 & 5.2 & 2.1 & 0.4 & 0.079 & 640 \\
N90 & 5 & -100 & 20 & 12.9 & 2.4 & 0.2 & 0.14 & 1.530 \\
N93 & 8 & -100 & 20 & 13.3 & 2.2 & 0.1 & 0.42 & 1.730 \\
& & & & Mean: & $2.3( \pm 0.05)$ & & \\
\hline
\end{tabular}

ing the test pulse (Armstrong and Bezanilla 1977). This agrees with the finding in the frog node of Ranvier, that "on" and "off" charge movements have the same voltage dependence (Dubois and Schneider 1982), indicating that both the immobilizable and the non-immobilizable gating charges have the same valence. As discussed later, the independence of $z_{a}$ on the number of allowed charge-carrying transitions suggests an approximately even distribution of the total gating charge between its most significant contributions.

The results of noise measurements performed on 14 different patches are summarized in Table 2. The $z_{a}$ estimates have a mean value of 2.3 and lie in the narrow range of 2.0 to 3.0 , while $\sigma_{B}^{2}$ and $Q$ vary by more than a factor of 10 due to variations in patch area and channel expression. The narrow range of $z_{a}$ suggests that major contaminations from recording artifacts are unlikely, because these are expected to produce excess variance which increases with the square of the signal rather than linearly with $I_{g}$ (see Eq. 4). Independent tests on membrane patches from non-injected oocytes showed neither appreciable gating currents nor the related excess noise. Likewise, oocytes with a poor expression of exogenous channels failed to produce a significant $I_{g}$ and any pulse-correlated change of membrane noise.

The hallmark of the shot-like character of the elementary contributions to the excess noise was the noncorrelation of fluctuations separated by more than the response time of the recording system. Figure 4 compares the autocovariance of the fluctuations centered at $0.5 \mathrm{~ms}$ before the test pulse with the autocovariance centered at the peak of the $I_{g}$ response. Both decay to negligible values with a time course dictated by the recording system and much faster than the $I_{g}$ response. Thus, at the highest time resolution that we could 
obtain in this study, gating-current fluctuations are indistinguishable from white noise, as expected from the superimposition of uncorrelated shot events. However, it should be stressed that by "shot event" we mean the movement of a finite charge packet having a maximum spread in time of about $25 \mu \mathrm{s}$.

\section{Discussion}

The voltage sensitivity of sodium channels must arise from large charge redistributions accompanying their structural transitions. From the voltage dependence of macroscopic sodium currents and gating-charge movements one can estimate the total charge, $z e_{0}$, which is displaced across the membrane in the transition of a single channel from the most favoured state at very negative potentials to the open state. In squid giant axons the transition of non-inactivated sodium channels, from their equilibrium state-distribution at $-40 \mathrm{mV}$ to the all-open configuration, is estimated to involve a mean displacement of 4 electron charges per channel, whereas step depolarizations from -120 to $-40 \mathrm{mV}$ produce about $1 / 3$ of the saturating levels of gating-charge movement (Stimers et al. 1985). This yields the estimate $z=6$ for squid sodium channels. The same argument applied to rat-brain-II sodium channels expressed in oocytes (e.g. Fig. 1 B) leads to $z$ estimates in the range of 6 to 8, with the uncertainty arising mostly from our inability to obtain both gating- and ionic-current data from the same oocyte.

The finding that the apparent single-gate charge estimated from noise data is about $1 / 3$ of the total gating-charge $\left(z_{a} \approx \mathrm{z} / 3\right)$ has important implications for the molecular mechanism of sodium channel gating. First, it provides direct evidence that a large portion of the gating charge moves by random steps that appear as discrete events on the time scale of normal electrophysiological processes. Furthermore, it confines the number and the size of the non-trivial components of $z e_{0}$ to narrower limits than those generally stated in connection with Eq. (5). Of great importance in this context is our additional finding that the same $z_{a} \approx z / 3$ accounts for both the "on" and the "off" gating-current fluctuations.

In most of our noise measurements the channels were fully inactivated at the end of the test pulse and only about $1 / 3$ of $z e_{0}$ appeared free to move back during the early phase of the "off" gating currents (Armstrong and Bezanilla 1977) (Fig. 2A). Since the apparent "off" single-gate is also about $1 / 3$ of $z e_{0}$, we are forced to conclude that $z_{a} \approx 2.3$ is the actual valence of the only significant charge movement which is not immobilized by inactivation. This implies in addition that the remaining (immobilizable) two thirds of $z e_{0}$ should contain at least two components (because they yield the same apparent valence of about $z / 3$ while they carry a total valence of $2 z / 3$ ), at least one of which has a valence of the order of $z / 3$. Indeed, the possible distributions of the immobilizable gating charge that satisfy Eq. (5) range between two extreme cases: a) one large component carrying a charge of about $\frac{\sqrt{2}}{3} z e_{0}$, combined with many very small additional components that account for the residual charge but do not yield any appreciable noise contribution; b) two major components carrying approximately the same charge $\approx \frac{1}{3} z e_{0}$. In any case, our results are inconsistent with models that assume a fragmentation of $z$ into a large number of contributions which are each on the order of or smaller than unity (Stimers et al. 1985).

The hypothesis that the total gating charge per channel is equally distributed between three major voltage-dependent steps provides the most simple explanation of our present noise data. Two further independent arguments support this hypothesis. First, the single-gate valence estimated from noise measurements is very close to the value of the parameter $z_{g}$ which yields the best fit of our $Q-V$ plots according to Eq. (6). That equation is consistent with the assumption that sodium channel activation is controlled by independent voltage-gated transitions which all involve the same gating charge $z_{g} e_{0}$ (Conti 1986). Furthermore, both $z_{a}$ and $z_{g}$ estimates agree closely with the $z_{m}$ that fits the voltage dependence of sodium activation according to Eq. (7), which implies a simple HH-type of activation scheme in which the opening of a sodium channel is preceded by three independent steps each producing the same transmembrane charge movement $z_{m} e_{0}$ (FitzHugh 1965). Within such scheme $z_{a}, z_{g}$ and $z_{m}$ are indeed different experimental estimates of the same physical quantity.

The consistency of our results with a model of sodium channel with three independent activation steps is not in contrast with the current notion that the classical HH-scheme is inadequate to describe the inactivation process and its coupling to activating. Such inadequacy is demonstrated by several results of gatingcurrent and single-channel measurements, most noticeably the dependence of the inactivating transition on the state of activation (Armstrong and Bezanilla 1977; Aldrich et al. 1983) and the immobilization of two thirds of the gating charge while the channel is in the inactivated state (Armstrong and Bezanilla 1977). However, none of these observations appear to contradict in any obvious way the hypothesis that the major voltage-sensitive activation steps occur independently of each other and involve approximately the same charge redistribution.

From our data we cannot rule out the possibility that the activating steps carrying an effective quantal 
charge movement of $2.3 e_{0}$ involve a single structural component undergoing a sequence of three independent successive rearrangements. However, it is more tempting to identify the gating-charge steps with structural transitions occurring in distinct pseudo-subunits of the sodium channel associated with three of the four repeats comprised by its primary structure (Noda et al. 1986) and to ascribe to the interaction of the activating subunits with the fourth a major role in inactivation. Despite its simplicity such model leaves room for many complications which can account for the failures of the HH-scheme. Indeed, besides inactivation other important but electrically silent transitions between different states of inter-subunit coupling may occur after the gating-charge steps. The actual opening of the channel-pore might in fact be one such transition, as suggested by the differential effects of $\mathrm{D}_{2} \mathrm{O}$ and pressure on $I_{a}$ or on the sodium currents (Conti and Palmieri 1968; Meves 1974; Schauf and Bullock 1979; Conti et al. 1984).

Acknowledgements. We are grateful to Prof. S. Numa and Dr. M. Noda for kindly making available to us the mRNA coding for rat-brain-II sodium channels. We thank E. Neher, B. Sakmann, F. Sigworth, and L. DeFelice for reading the manuscript and for helpful discussions. F. C. is a Scholar-in-Residence of the Fogarty International Center, National Institutes of Health, Bethesda, MD, USA.

\section{References}

Aldrich RW, Corey DP, Stevens CF (1983) A reinterpretation of mammalian sodium channel gating based on single channel recording. Nature 306:436-441

Armstrong CM, Bezanilla F (1974) Charge movement associated with the opening and closing of the activation gates of the $\mathrm{Na}$ channels. J Gen Physiol 63:533-552

Armstrong CM, Bezanilla F (1977) Inactivation of the sodium channel. II. Gating current experiments. J Gen Physiol $70: 567-590$

Conti F (1986) The relationship betwen electrophysiological data and thermodynamics of ion channel conformations. Neurol Neurobiol 20:25-41

Conti F, Stühmer W (1988) Gating currents and their fluctuations in sodium channels expressed in Xenopus oocytes after injection of exogenous mRNA. $11^{\text {th }}$ Annual Meeting of the European Neuroscience Association

Conti F, Palmieri G (1968) Nerve fiber behaviour in heavy water under voltage-clamp. Biophysik 5:71-77

Conti F, Wanke $E$ (1975) Channel noise in nerve membranes and lipid bilayers. Q Rev Biophys 8:451-506

Conti F, DeFelice LJ, Wanke E (1975) Potassium and sodium ion current noise in the membrane of the squid giant axon. J Physiol (London) 248:45-82

Conti F, Neumcke B, Nonner W, Stämpfli R (1980) Conductance fluctuations from the inactivation process of sodium channels in myelinated nerve fibres. J Physiol (London) 308: $217-239$

Conti F, Inoue I, Kukita F, Stühmer W (1984) Pressure dependence of sodium gating currents in the squid giant axon. Eur Biophys J 11:137-147
DeFelice LJ (1981) Introduction to membrane noise. Plenum Press, New York

Dubois JM, Schneider MF (1982) Kinetics of intramembrane charge movement and sodium current in frog node of Ranvier. J Gen Physiol 79:571-602

Edmonds DT (1987) A comparison of sodium channel kinetics in the squid axon, the frog node and the frog node with BTX using the "silent gate" model. Eur Biophys J 15:27-33

FitzHugh R (1965) A kinetic model of the conductance changes in nerve membrane. J Cell Comp Physiol 66:111-118

French RJ, Horn R (1983) Sodium channel gating: models, mimics, and modifiers. Annu Rev Biophys Bioeng 12:319-356

Hamill OP, Marty A, Neher E, Sakmann B, Sigworth FJ (1981) Improved patch-clamp techniques for high resolution current recording from cells and cell-free membrane patches. Pflügers Arch 391:85-100

Hodgkin AL, Huxley AF (1952) A quantitative description of membrane current and its application to conduction and excitation in nerve. J Physiol (London) 117:500-544

Horn R, Vandenberg CA (1984) Statistical properties of single sodium channels. J Gen Physiol 84:505-534

Katz B, Miledi R (1970) Membrane noise produced by acetylcholine. Nature 226:962-963

Keynes RD (1983) Voltage-gated ion channels in the nerve membrane. Proc R Soc Lond B220:1-30

Keynes RD, Rojas E (1976) The temporal and steady-state relationships between activation of the sodium conductance and movement of the gating particles in the squid giant axon. J Physiol 255:157-189

Methfessel C, Witzemann V, Takahashi T, Mishina M, Numa S, Sakmann B (1986) Patch clamp measurements on Xenopus laevis oocytes: currents through endogenous channels and implanted acetylcholine receptor and sodium channels. Pflügers Arch 407:577-588

Meves $H$ (1974) The effect of holding potential on the asymmetry currents in squid giant axons. J Physiol (London) 243: $847-867$

Neher E, Sakmann B (1976) Single-channel currents recorded from membrane of denervated frog muscle fibres. Nature 260:799-802

Neher E, Stevens CA (1977) Conductance fluctuation and ionic pores in membranes. Annu Rev Biophys Bioeng 6:345-381

Neumcke B, Nonner W, Stämpfli R (1976) Asymmetrical displacement current and its relation of the activation of sodium current in the membrane of frog myelinated nerve. Pflügers Arch 363:193-203

Noda M, Ikeda T, Suzuki H, Takeshima H, Kuno M, Numa S (1986) Expression of functional sodium channels from cloned cDNA. Nature 322:826-828

Schauf CL, Bullock JO (1979) Modifications of sodium channel gating in Myxicola giant axons by deuterium oxide, temperature, and internal cations. Biophys J 27:193-208

Schottky W (1918) Über spontane Stromschwankungen in verschiedenen Elektrizitätsleitern. Ann Phys 57:541-567

Schwarz G (1978) On the physico-chemical basis of voltage-dependent molecular gating mechanisms in biological membranes. J Membr Biol 43:127-148

Sigworth FJ (1977) Sodium channels in nerve apparently have two conductance states. Nature 270:265-267

Stimers JR, Bezanilla F, Taylor RE (1985) Sodium channel activation in the squid giant axon. Steady state properties. J Gen Physiol 85:65-82

Stühmer W, Methfessel C, Sakmann B, Noda M, Numa S (1987) Patch clamp characterization of sodium channels expressed from rat brain cDNA. Eur Biophys J 14:131-138

Stühmer W, Conti F, Suzuki H, Wang X, Noda M, Yahagi N, Kubo H, Numa S (1989) Structural parts involved in activation and inactivation of the sodium channel. Nature (in press) 